\title{
Dynamic Recrystallization in a Naturally Deformed Albite
}

\author{
J. D. FITZ GERALD†§, M. A. ETHERIDGE† and R. H. VERNON $\ddagger$
}

†Department of Earth Sciences, Monash University, Clayton, Victoria 3168, Australia

$\ddagger$ School of Earth Sciences, Macquarie University, North Ryde, N.S.W. 2113, Australia

$\S$ Now at: Research School of Earth Sciences, Australian National University, Canberra, A.C.T. 2601, Australia

(Received May 8, 1982; in final form November 19, 1982)

Coarse-grained, deformed albite occurs in veins within a blueschist from the Cazadero region, California. In some grains, deformation and recrystallization are concentrated in narrow shear zones less than $50 \mu \mathrm{m}$ wide. We have examined the substructural progression across these zones by transmission electron microscopy (TEM), in an attempt to determine the details of the dynamic recrystallization mechanism. The misorientation across subgrain and recrystallized grain boundaries has been determined by analysis of electron diffraction patterns.

Dynamic recrystallization apparently proceeded by the following stages: 1) the formation of a well-ordered substructure from a more tangled, cell-like array, 2) increasing misorientation between subgrains, 3 ) rapid growth of subgrains at a misorientation between $3^{\circ}$ and $5^{\circ}$ to produce new "grains" with straighter grain boundaries and lower internal dislocation densities and 4) continued deformation and rotation of the recrystallized grains with local grain-boundary migration to maintain relatively equiaxed shapes. The ultimate recrystallized structure in the narrow deformation zones consists of grains misoriented by between $5^{\circ}$ and at least $30^{\circ}$, most of them containing a well-developed substructure.

The combination of subgrain growth and rotation explains a number of features common to dynamically recrystallized minerals. The smaller subgrains present prior to growth and also within recrystallized grains form a population distinct from the larger subgrains and recrystallized grains of approximately equal size, which are those observed in an optical microscope. The smaller subgrains are visible only in TEM. Individual recrystallized grains may remain through substantial straining, rotating in response to dislocation and sub-boundary motion within them, thus preserving and even enhancing the crystallographic fabric (texture). The retention of an initial recrystallized grain population throughout significant continuing deformation may explain the absence of strain softening in some recent experimental studies.

\section{INTRODUCTION}

High-temperature creep of metals and ceramics results in a stable microstructure that generally consists of an array of cells or subgrains, the size 
and relative misorientation of which remain constant throughout deformation. In some metals and many minerals, however, the misorientation between subgrains increases with creep strain, eventually resulting in substantial recrystallization. This mechanism of dynamic recrystallization has become known as subgrain rotation, and has been widely recognized in quartz (Hobbs, 1968; White, 1973, 1976, 1977; Bell and Etheridge, 1976), olivine (Poirier and Nicolas, 1975), pyroxene (Etheridge and Kirby, 1983), calcite (Schmid et al., 1980) and feldspar (White, 1975).

Much of the evidence for this mechanism comes from optical and qualitative observations in the transmission electron microscope (TEM), and there is very little information on the details of the changes in dislocation substructure. In particular, there has been no correlation between TEM measurements of misorientation between subgrains and changes in boundary substructure. Without this information, the role of dynamic recrystallization in creep (e.g. leading to grain boundary sliding and superplastic behaviour via grainsize reduction), and the relationship between recrystallized grainsize and creep stress (Edward et al., 1982) cannot be understood.

This paper describes the optical and TEM substructure of a partly recrystallized pure albite, including measurements of the misorientation across a range of sub-boundaries and grain boundaries. Misorientations were computed from selected-area electron diffraction patterns of adjacent parts of the substructure. This albite, from the Franciscan Formation, Cazadero, California, was chosen because: 1) it contains zones, some shear-related, in which there is a transition from a weakly deformed to a recrystallized microstructure over only tens of microns, and 2) the pure albite composition ensures that the driving force for recrystallization is derived entirely from the stored strain energy, without the chemical free-energy component that complicates the recrystallization process in other feldspars (White 1975; Marshall et al., 1976).

The albite occurs in coarse-grained, milky white veins in a blueschist, the assemblage of which is glaucophane + omphacitic pyroxene + epidote + white mica + sphene + minor albite. Apart from the albite, this assemblage belongs to the type IV metabasalts of Coleman and Lee (1963). The relationship between the albite veins and the metamorphism is not clear, but the maximum temperature defined by the assemblage is $500^{\circ} \mathrm{C}$ (Taylor and Coleman, 1968; Ernst, 1973, p. 258), which is therefore the maximum temperature for the deformation of the albite.

\section{OPTICAL MICROSTRUCTURE}

Grains within the albite veins are between 1 and $5 \mathrm{~mm}$ across, and most 


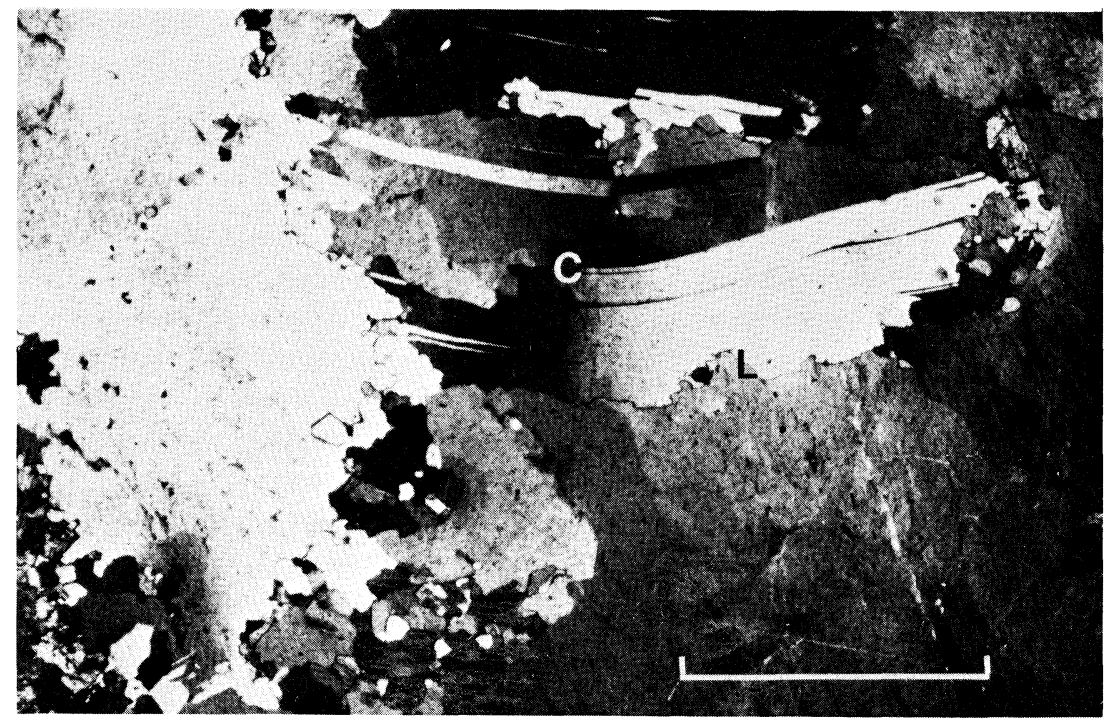

FIGURE 1 Curved twin boundaries (C) and lobate albite-albite grain boundaries (L) in coarse-grained Cazadero albite. Minor recrystallization has also taken place in grain boundary regions. (Optical micrograph, crossed polars, scale bar $=0.5 \mathrm{~mm}$ ).

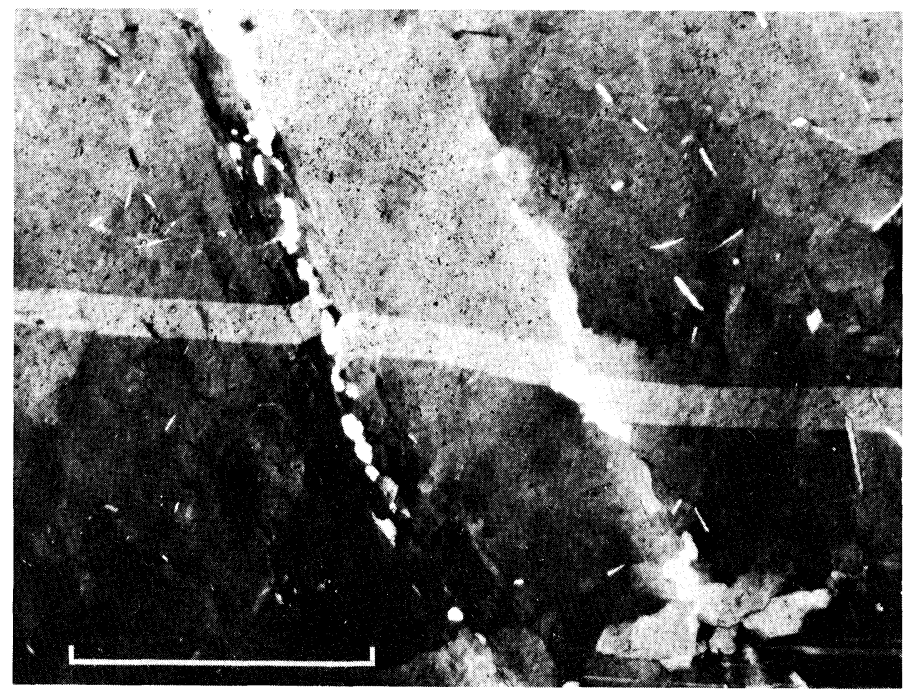

FIGURE 2 Narrow $(20-100 \mu \mathrm{m})$ planar deformation zones, within a large albite grain, across which an isolated albite-law growth twin has been offset. These zones are characterized by subgrain development and recrystallization, and are typical of the sites selected for the detailed TEM work described herein. (Optical micrograph, crossed polars, scale bar $=0.5 \mathrm{~mm}$ ). 
contain several albite-law growth twins that are typically 50-1000 $\mu$ m wide and traverse the entire grain. Interlobate albite-albite grain boundaries and twin boundaries (Figure 1) indicate that strain-induced grain boundary migration has operated over wavelengths of $30 \mu \mathrm{m}$ and larger. Both curved and planar segments occur along bulged boundaries.

The weak deformation structure present throughout most grains (Figure 2) involves slightly misoriented, elongate, blocky cells (ca. $10 \times 50 \mu \mathrm{m}$ ). Locally, increasing deformation is accompanied by pronounced optical misorientation and concentrations of smaller, more equant subgrains and new grains. The smallest new grains are $2-10 \mu \mathrm{m}$ across, but new grain sizes in extensively recrystallized zones reach $300 \mu \mathrm{m}$, indicating substantial grain growth. The shapes of new grain boundaries range from planar to curved.

Growth twins maintained simple boundary shapes during deformation and are useful indicators of strain. The twins are commonly bent in smooth, continuous shapes with deflections of up to $30^{\circ}$ (Figure 1). Depending on the width of the zone, shear strains of up to 0.5 are attained. Alternatively, the twins are markedly offset by up to $50 \mu \mathrm{m}$ on cross-cutting planar displacement zones (Figure 2). The major component of displacement is restricted to a narrow $(10-30 \mu \mathrm{m})$ band with minimum values of shear strain between 2 and 3, calculated from displacements across the bands. In places, local curvatures of twin planes provide evidence for flanking zones of weak deformation up to $50 \mu \mathrm{m}$ from the main displacement. The sense of curvature of twins in this flanking zone is generally, but not necessarily, consistent with the sense of displacement across the central zone. Planar displacement features may pass into narrow zones of incipient recrystallization or into small numbers of subgrains (Figure 2) and new grains.

In a few places, concentrations of discontinuous albite-law twins, 1-5 $\mu \mathrm{m}$ wide, are intimately associated with zones of increased deformation. In less deformed parts of these zones, groups of twins terminate against (100) walls of weakly misoriented cells. Such a relationship requires that these be deformation twins (Vogel and Seifert, 1965).

\section{TRANSMISSION ELECTRON MICROSTRUCTURE}

Nine specimens selected from three optical thin sections were ion-beam thinned and examined with a JEM200A electron microscope fitted with a goniometer stage and operated at $200 \mathrm{kV}$. In ion-thinned specimens, foil thickness increases sharply away from the perforated edge, so that most regions transparent to $200 \mathrm{kV}$ electrons are within a few tens of microns of the specimen edge. Such a distribution of transparent regions is suitable for examination of specimen detail at all scales smaller than a few tens of microns, 
but creates some difficulty in assessment of the coarser microstructures (e.g. blocky cell structure and coarse new grains) in the Cazadero albite.

\section{Dislocation substructure in weakly deformed regions}

In weakly deformed parts of the albite, unbound dislocations are always present and generally widely spaced, so that interactions are limited to minor tangles. Dislocation lines show no distinct preferred direction, but locally are straight and aligned parallel to [001] (Figure 3). TEM images from thin foils oriented normal to [100] commonly feature an orthogonal pattern, due to the predominance of dislocation lines in $(010)$ and $(001)$ planes.

Some regions of the foils feature planar defects parallel to (010) which may extend for several microns and terminate at dislocations oriented near to [001]. These defects have been identified as albite-law microtwins by analysis

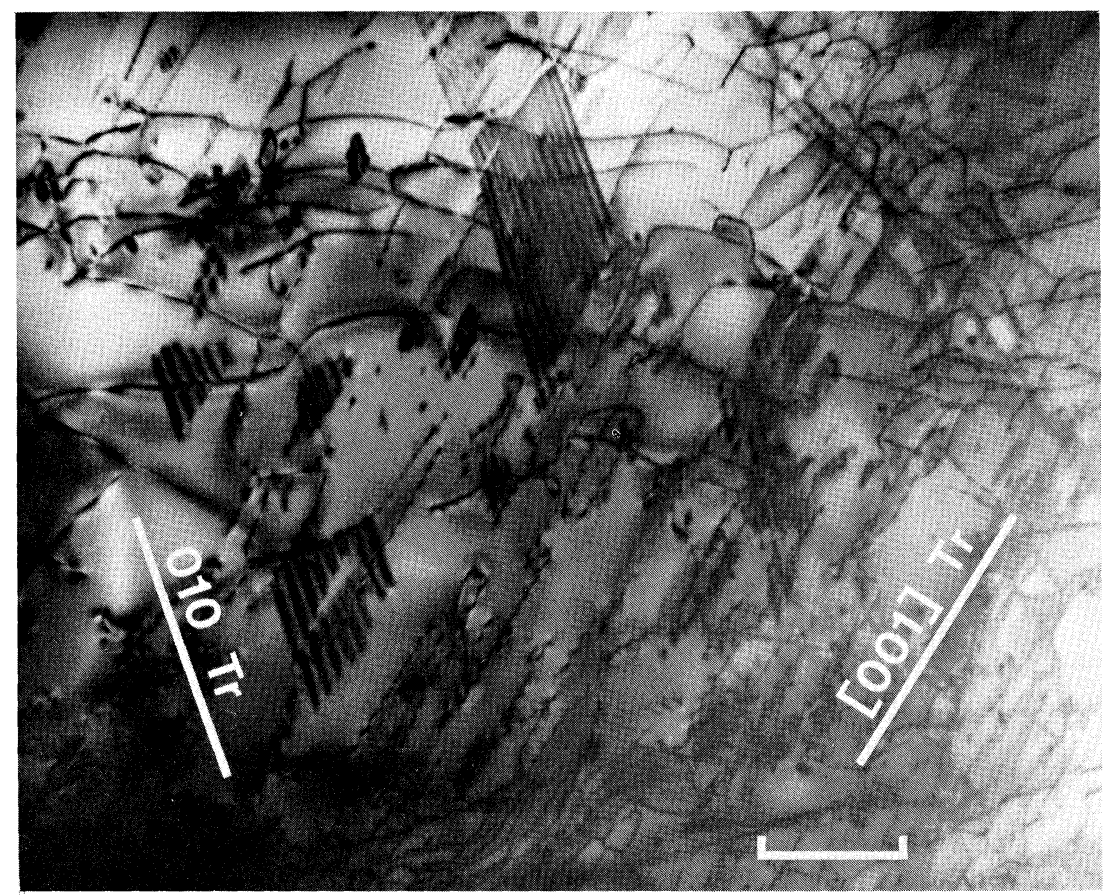

FIGURE 3 Bright field electron micrograph of weakly deformed region in Cazadero albite, with beam axis near [112]. Microtwins are inclined to this axis and image as fringe patterns with their trace parallel to $(010)$. Many microtwin terminations and unbound dislocations lie parallel to [001]. Scale bar $=1$ micron. 
of bright- and dark-field fringe contrast. The thickness of each microtwin is constant and corresponds to a small integer multiple (commonly two) of unit cells. (Fitz Gerald, 1983, in prep.). Many dislocations adjacent to albitelaw microtwins are parallel, like the microtwin terminations, to [001], and some are twinning dislocations (Figure 3).

Unique determination of Burgers vectors has not been attempted. Invisibility criteria are likely to produce ambiguous results due to the elastic anisotropy of feldspars (Marshall and McLaren, 1977), and the lack of appropriate elastic constants precludes any rigorous computed simulation of dislocation images in triclinic feldspar structures. However, orientations of non-overlapping slip traces (Marshall and McLaren, 1977) have been estimated in regions of low dislocation density. Some dislocation lines subparallel to [001] feature curved slip traces near (010). The non-planar traces may be identified with cross-slip of [001] screw dislocations, simultaneous glide and climb of almost pure edge dislocations with $b=$ [201], or complex motions of mixed dislocations. Application of invisibility criteria, using diffracting conditions such that $g=020$ or 110 , suggests that $b=[001]$ is the most appropriate choice. This Burgers vector is the shortest available for $\bar{C} \overline{1}$ plagioclases, but has not been observed widely in experimentally deformed feldspars (Marshall and McLaren, 1977), or in albite naturally deformed at upper greenschist facies conditions (Marshall and Wilson, 1976). However, it was the dislocation most commonly identified in low-An plagioclases, naturally deformed at granulite grade, examined by Olsen and Kohlstedt (1981).

Planar to curved dislocation arrays are commonly present (Figures 4a, b). They contain relatively low densities of dislocations, which may be either loosely tangled or in well-knitted, regular networks. The more loosely tangled arrays generally occur in areas of low strain and poorly developed substructure, whereas the regular networks are more commonly associated with recrystallized regions. The arrays are continuous, and locally occur in sets of particular orientations and spacings. Intersection of two or more sets outlines a blocky cell structure (Figure $4 \mathrm{~b}$ ) on a scale similar to that described previously from optical observations.

The orientation and regularity of cell walls vary widely. Although (010), (001), (110) and (110) walls are present, many walls are not constrained to low-index crystallographic orientations, and they are commonly non-planar (compare Figures 4a, b). Pronounced changes in wall spacing and orientation (i.e. dominance of particular sets of dislocation walls) are observed across distances of tens of microns. It follows that cell wall construction varies considerably, even between adjacent regions. Isolated, rounded cells are present, but are rare compared to cells formed by wall intersections. These independent cells may be termed true subgrains, as dislocations in their boundaries are 


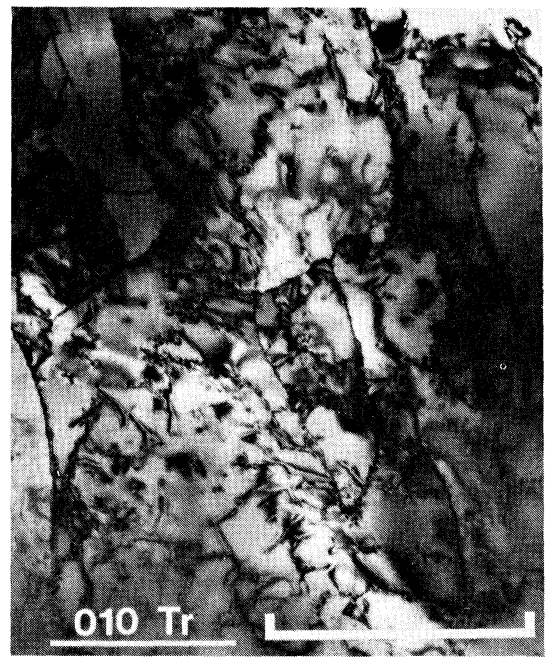

a

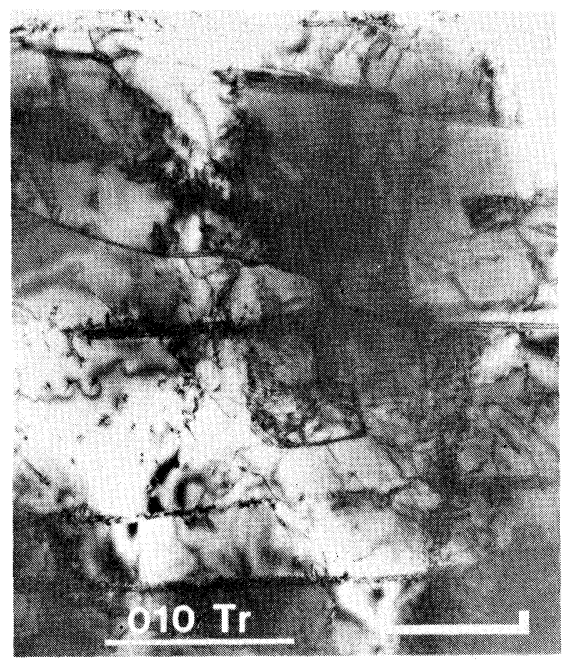

b

FIGURE 4 Dislocation substructure in weakly deformed regions of albite. The two regions pictured are only about 30 microns apart. (a) Irregularly shaped cells and dislocation walls; (b) Straight dislocation walls aligned sub-parallel to (010) and (100). Bright field images, beam axis near [001], scale bar $=5$ microns.

commonly arranged in networks to the exclusion of tangles (which are more characteristic of cell walls).

Voids smaller than $0.1 \mu \mathrm{m}$ in diameter are locally abundant and commonly have "negative-crystal" shapes. They lie in dislocation walls, with densities of 1-2 voids per cell. The low average concentration and sporadic occurrence of voids indicate that they were of little importance in the overall deformation.

\section{Sequence of recrystallization}

In TEM investigations, sharp changes in deformation substructure over distances of the order of $30 \mu \mathrm{m}$ have been correlated with optical observations in the edges of deformed zones. The following sequence is consistently observed with increasing strain in these zones (Figure 5a).

i) The substructure of the weakly deformed bulk of the material, as already described, consists of large, elongate cells (ca. $10 \mu \mathrm{m}$ ) with both dislocation networks and tangles constituting cell walls and low to moderate densities of internal, unbound dislocations.

ii) Higher densities of unbound dislocations are associated with increasing strain. Dislocations are forced to interact within the cells to form loose 


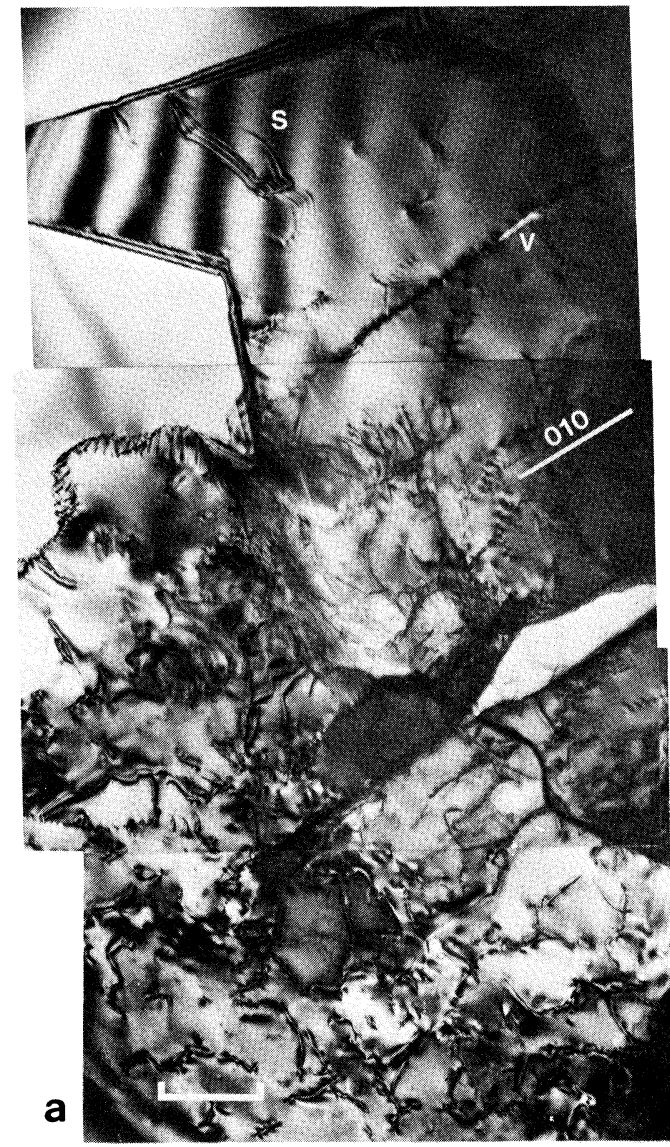

(v)

(iv)

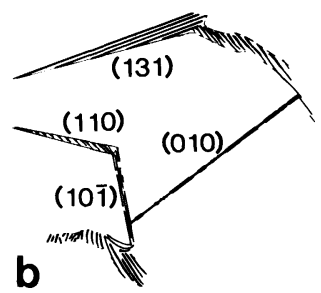

(iii)

(i)-(ii)

FIGURE 5 Sharp transitions in deformation substructure across narrow deformation zones in albite. Stages (i) to (v) marked on the figures are described in the text. (a) Bright field micrograph with beam axis near $[\overline{10} \overline{2}]$ using the $(0 \mathrm{k} 0)$ row. Note the slip plane traces $(\mathrm{S})$ in low dislocation density regions, and the voids (V) along grain boundaries. Many of the dislocations lying in (010) are out of contrast. Doubled dislocation images are due to excitation of more than one diffracted beam. Scale bar $=1$ micron. (b) Sketch showing orientations of the planar segments in stage (v) of Figure 5a.

tangles or high-density, tangled dislocations in walls of finite width and irregular shape.

iii) Intersections of walls of different orientations produce small (1-3 $\mu \mathrm{m})$, generally elongate, blocky cells. Cell walls consist dominantly of tight dislocation tangles, but ordered dislocation networks are also present. Unbound dislocations persist to varying degrees within these small cells. iv) Continuation of the process involves development of ordered walls to 
the exclusion of tangled walls. In general, cells with ordered walls are larger, and their internal dislocations aggregate into loose networks (as opposed to tangles). The narrow, ordered walls tend to have gently curving shapes, and to define relatively equant cells, which could properly be termed subgrains.

v) The final step is a sharp change to larger (5-10 $\mu \mathrm{m})$ equant grains, mostly free of unbound dislocations. Grain walls are very regular and commonly parallel to low-index crystallographic planes (Figures 5a, b). Many of the TEM images include substantial densities of dislocations in grain walls, and the distinction between "low" and "high" angle boundaries is not clear. However, there is a distinct change in the character of dislocation walls, presumably associated with an increase in misorientation and in boundary energy, as would be expected for recrystallized grains.

In the more highly deformed areas, highly misoriented new grains

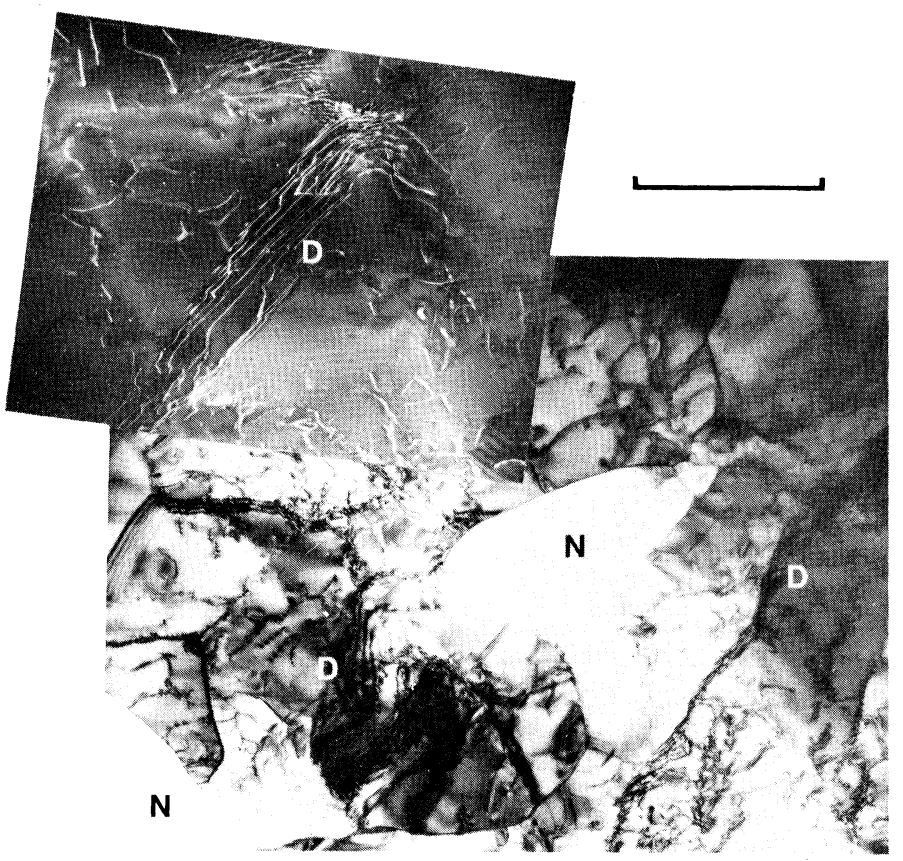

FIGURE 6 Dislocation structures near isolated new grains. Composite bright and dark field micrograph. The frame in the upper left is a weak-beam dark field $(g=040)$ image while the lower right is a bright field photograph using complementary diffraction conditions (beam axis near [001], (020) runs approximately parallel to the shorter edge of the lower right photograph). Isolated high-angle new grains $(\mathrm{N})$ reside in a matrix showing dislocation wall structures (D) characteristic of weak to moderate deformation. Scale bar $=5$ microns. 
(measured rotations greater than ca. $8^{\circ}$ ) are characteristically enclosed by dislocation-free, straight to curved boundaries, commonly preferentially etched during ion thinning, and are generally adjacent to regions of welldeveloped dislocation structure (but see below). The minimum size of new grains observed in each area is commonly 3-5 $\mu \mathrm{m}$, with grains of this size tending to cluster together. Large new grains were difficult to observe in TEM, being recognized only as segments of extensive, curved to planar grain boundaries with misorientations of $10-40^{\circ}$. Dislocation densities within new grains are quite variable, but the dislocations adopt the configurations already described for progressive deformation. The structure within larger new grains has progressed at least to an intersecting tangled wall and network stage.

Highly misoriented grains are not always adjacent to regions of advanced substructure development. They have occasionally been observed in regions showing typical stage (ii) microstructures, accompanied by a weak tendency for dislocations in the walls to be organized into networks (Figure 6).

\section{Misorientation measurements}

Measurements have been made of the misorientation across a range of cell walls and boundaries. Strong, sharp Kikuchi line patterns were not well developed by these deformed silicate mineral foils; therefore accurate misorientation parameters could not be determined using metallographic methods such as those of Young et al. (1973), Faivre (1975) or Clarebrough and Forwood (1980). Furthermore, the small coherent grainsizes of the albite foils prohibited orientation analysis via simple selected area diffraction, as in the method of Pumphrey and Bowkett (1970). Instead, a procedure was devised by which the orientations of small regions could be defined using electron diffraction patterns together with intensities in bright and dark field images observed during controlled tilting experiments. Subsequent calculation of misorientations between regions (i.e. across walls and boundaries) is straightforward in theory. As the results of this tilting method are somewhat imprecise and the procedure most time consuming, relatively few measurements were made during this investigation. However, the following general conclusions can be drawn from the areas analysed, three of which are detailed in Figures 7, 8a and $b$.

i) The correlation between boundary appearance and misorientation is not good for misorientations greater than $1^{\circ}$ or $2^{\circ}$. Some straight, sharp boundary images correspond to very small misorientations (e.g. $1.6^{\circ}$ for lower left boundary in Figure 7), whereas higher angle boundaries may show quite complex dislocation structure (e.g. $6.1^{\circ}$ and $6.4^{\circ}$ boundaries in centre of Figure 7). The boundary image depends, of course, on its 


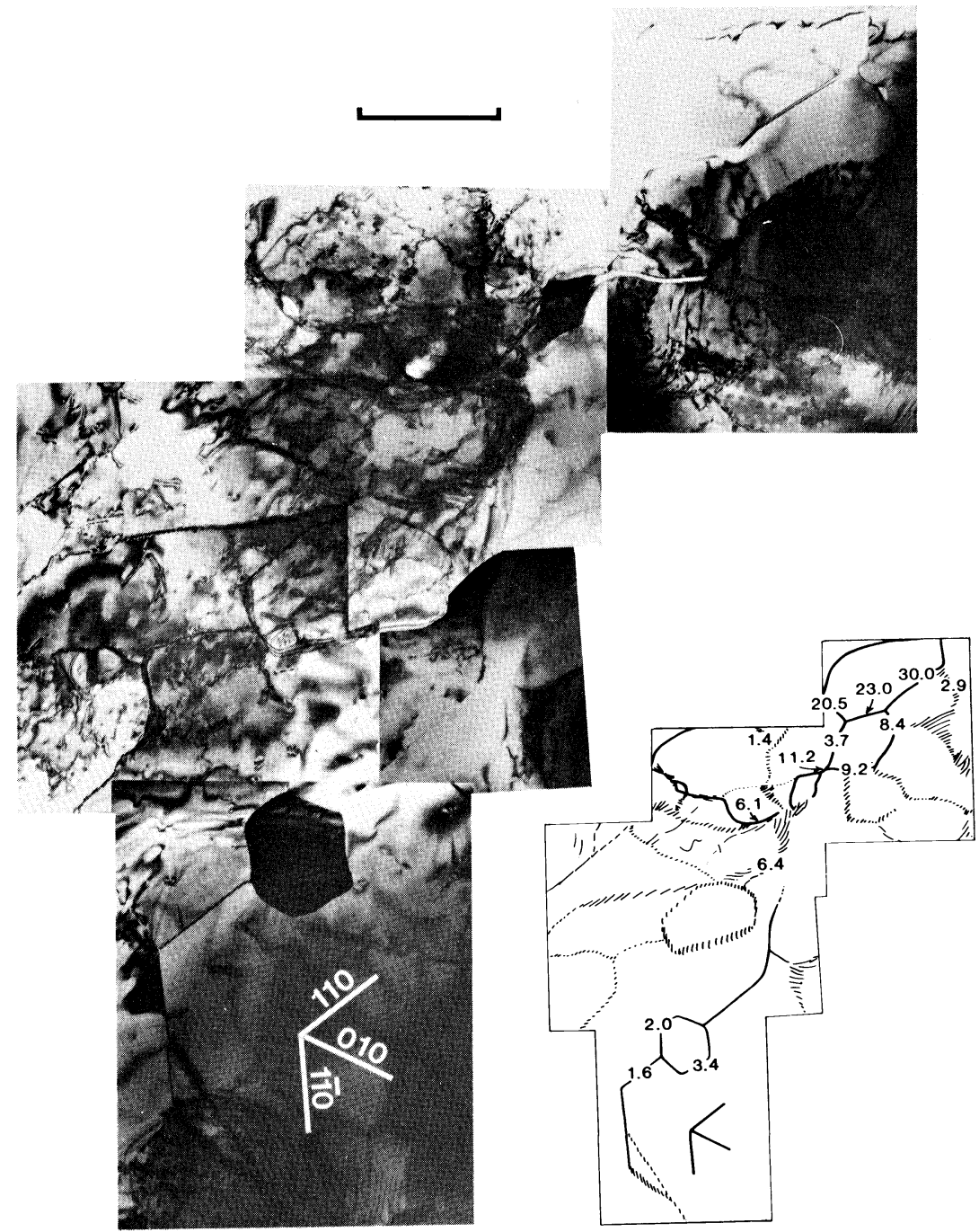

FIGURE 7 Bright field composite micrograph showing variation in dislocation and new grain structures. Measured misorientation angles are indicated in the adjacent sketch. Boundary appearance and degree of misorientation are not well correlated. Note the highly misoriented grains adjacent to unrecrystallized material in the upper right of the photograph. Speckled contrast due to beam damage. Scale bar $=5$ microns.

orientation with respect to the beam axis, those boundaries in the same zone as the beam appearing to be sharp, irrespective of structure, and 

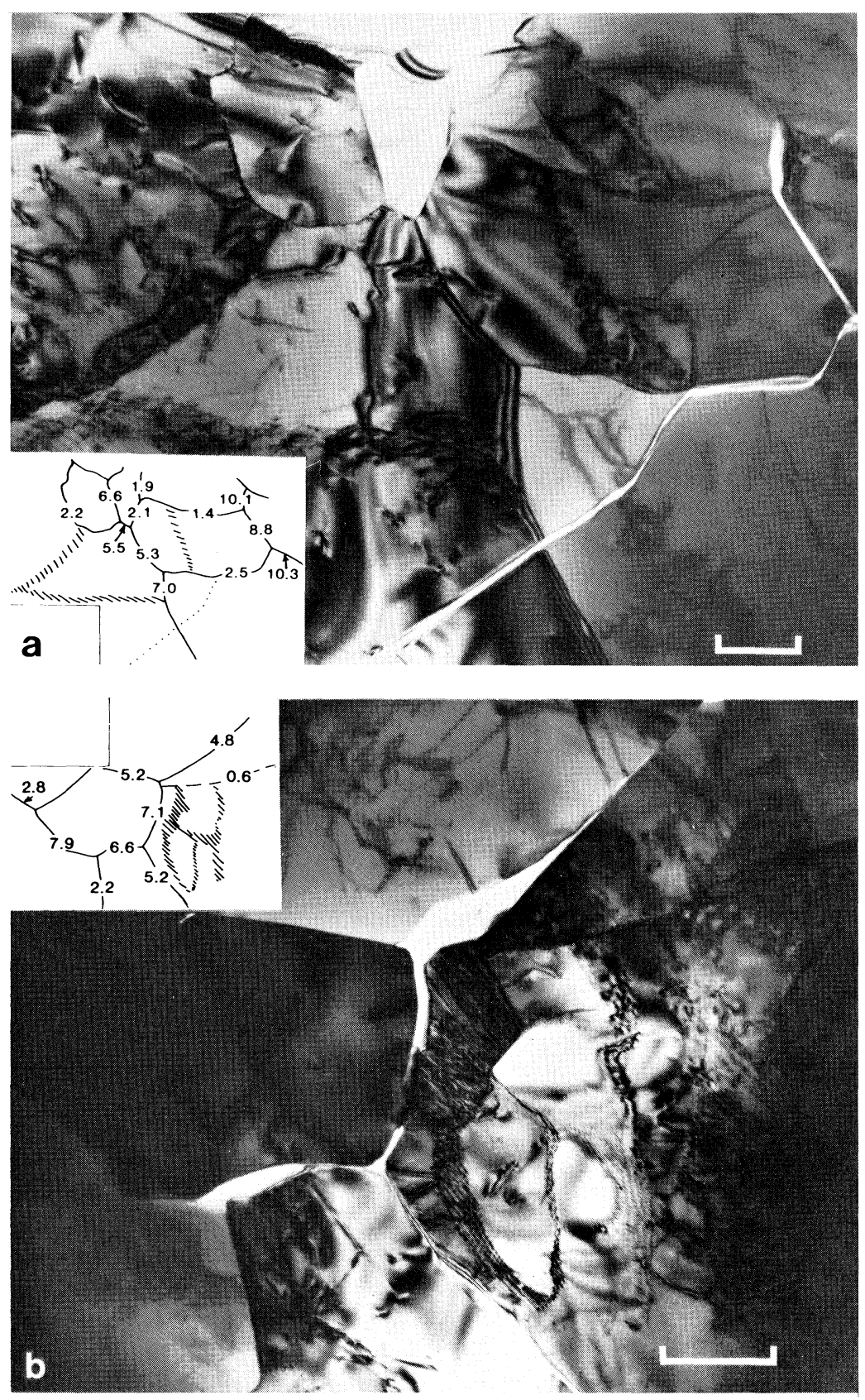

FIGURE 8 New grain aggregates with measured misorientations shown on the accompanying sketches. (a) Bright field image with beam axis near [001]. (b) Bright field image with beam axis near [112], note ion-etching of higher angle boundaries. Scale bars $=1$ micron. 

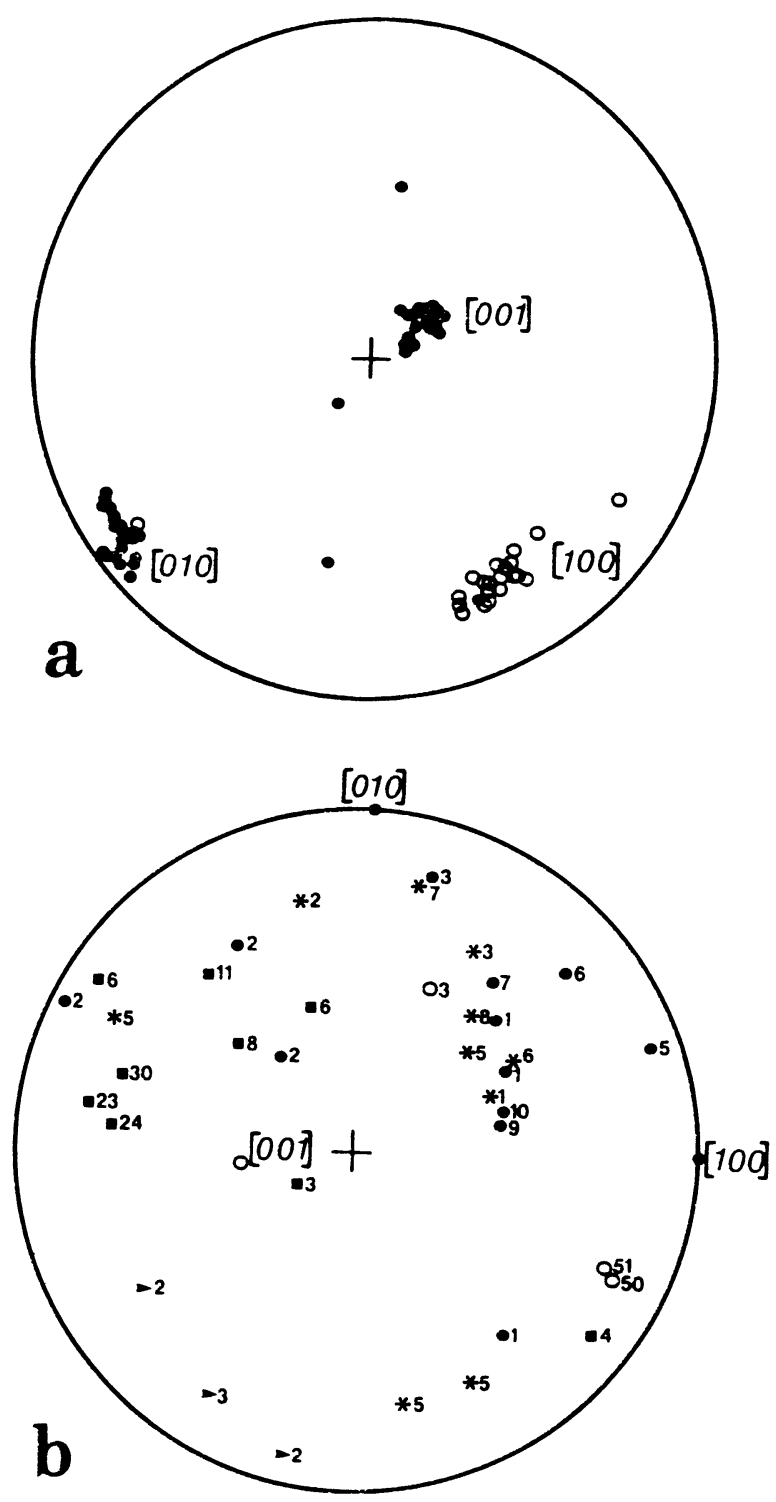

FIGURE 9 (a) Equal area stereographic projections of orientations of grains measured in Figures 7 and 8 . The foil normal is vertical, and the foil was cut approximately perpendicular to [001]. Closed symbols $\equiv$ lower hemisphere; open symbols $\equiv$ upper hemisphere.

(b) Equal area stereographic projection (upper hemisphere) of rotation axes between adjacent grains in Figures 7 and 8. Axes are plotted with respect to albite unit cell axes, and rotation angles (misorientations) are given in degrees. Axes with the same symbol are from small, contiguous groups of grains. 
vice versa. However, real variation of boundary structure was found here not to correlate with misorientation.

ii) The simple progression in microstructure described above (e.g. Figure 5a) is not necessarily mimicked by a similar progression in boundary misorientation (Figure 7).

iii) The highest misorientations measured occur around isolated recrystallized grains in relatively weakly deformed regions (top right of Figure 7; one other example is plotted in Figure $9 \mathrm{~b}$, but not pictured). In thoroughly recrystallized regions, most misorientations are in the range: $5^{\circ}$ to $10^{\circ}$.

iv) The computed axes of rotation for the boundaries shown in Figures 7 and 8 are plotted in an equal-area projection in Figure 9. They show some tendency to cluster around particular crystallographic directions, but, without precise information on operative slip systems, it is impossible to compare sub- and new grain rotation axes with rotations due to slip in the original albite crystal. The strong crystallographic preferred orientation of the measured recrystallized grains (Figure 9a) is to be expected from a cluster of grains with small relative misorientations.

\section{DISCUSSION}

The microstructural transition with increasing strain-the early stages

We have described a relatively sharp microstructural transition from weakly to strongly deformed albite across narrow regions with large strain gradients. In the following discussion, it is assumed that this transition is equivalent to the progressive development of the most highly strained microstructures.

Stages (i)-(iii) of the microstructural transition described above are typical of the hardening stage that most materials undergo at high temperature, prior to steady state deformation (McQueen and Jonas, 1975; Takeuchi and Argon, 1976; White, 1977). The initial rapid increase in dislocation density gives rise to the high primary creep rate (or the microstrain interval of hot working described by McQueen and Jonas, 1975), but the resulting dislocation interactions lead to subsequent hardening. Only when a stable, well-ordered substructure develops is secondary (steady-state) creep achieved. In many materials (especially those with high stacking fault energies), very large strains can be achieved without change to this stable substructure, and certain microstructural parameters (e.g. dislocation density and subgrain size) are a function of the creep stress. However, the well-ordered subgrain structure in albite 
is, as in many other minerals, merely a stage in the progression to a dynamically recrystallized microstructure.

\section{The nucleation stage}

The progression to dynamic recrystallization also takes place in some metals (McQueen and Jonas, 1975; Sellars, 1978), but nucleation is almost always associated with pre-existing high-angle boundaries in polycrystals (the bulge nucleation of Bailey and Hirsch, 1962). The phenomenon of subgrain rotation, whereby the misorientation across sub-boundaries increases with strain until high-angle boundaries are formed, was first alluded to by Hobbs (1968), and has been widely inferred (largely from optical observations) for a number of minerals (White, 1973, 1977; Poirier and Nicolas, 1975; Bell and Etheridge, 1976; Schmid et al., 1980; Etheridge and Kirby, 1983). It has been concluded that dislocations enter sub-boundaries faster than they can be annihilated during straining, giving rise to progressively higher boundary misorientations. However, the precise mechanism by which this rotation and change in boundary character takes place is unknown.

In the Cazadero albite, the change from a classical subgrain structure (as in stage (iv) described above) to "grains" with higher-angle, straighter boundaries is sudden. It is also accompanied by a twofold increase in grainsize and a marked reduction in free dislocation density. The boundary misorientation at which this transition takes place is anywhere between $3^{\circ}$ and $5^{\circ}$. Clearly, this sudden transformation in the substructure did not solely involve the progressive increase in dislocation density and thus misorientation of pre-existing sub-boundaries. The increase in grainsize must have involved either growth of individual subgrains at the expense of their neighbours, or coalescence of two or more adjacent subgrains. The change in shape of the boundaries implies an increase in their mobility, with straightening presumably being a response to their increase in energy with higher misorientation. Finally, the marked reduction in free dislocation density can only be satisfactorily explained by absorption of dislocations in highly mobile boundaries that swept through most of the local crystal volume at this stage. This grain boundary migration must have been rapid compared to the generation rate of dislocations, and supports the notion of subgrain growth rather than coalescence. However, the growth stage was just as suddenly arrested, giving rise to a relatively stable dynamically recrystallized grainsize, within which free dislocations and loosely knitted arrays developed with further straining.

What initiated and drove this growth stage, and why was it arrested? Grain boundary mobility is known to increase with misorientation (Aust and Rutter, 1959; Gordon and Vandemeer, 1966), neglecting the stress-driven glide of low-angle tilt boundaries, and there is commonly a critical angle at which 
it increases suddenly. In Cazadero albite, the grain growth was driven by the strain energy resulting from the free dislocations within subgrains, and by the boundary energy itself as the boundary area decreased. The approximately twofold increase in grainsize was sufficient to effectively clear the "grains" of dislocations and remove the first of these driving forces. Let us assume that this was the major driving force, and that its removal substantially slowed grain growth. Continuing deformation would have generated more dislocations within the larger grains. These dislocations knitted into subboundaries, because the stable subgrain size was less than the grainsize. Any tendency to further increase the grainsize was in competition with the formation of new boundaries, and the balance between these competing processes controlled the steady-state microstructure and the creep process.

The nucleation mechanism outlined above is, in some ways, intermediate between those presented by White (1977, Figure 3), in that both subgrain rotation and grain boundary migration are involved. White suggested that the two mechanisms represent different balances between recovery rate and work hardening rate, with boundary migration favoured where the hardening rate locally exceeds recovery rate. However, we propose that the grain boundary migration (growth) step is a fundamental part of what Guillopé and Poirier (1979) termed "rotation recrystallization", since recrystallized grains are larger than subgrains in all materials so far examined (see Twiss, 1977, Figure 1 for a compilation). The subgrains referred to here are those observed by TEM; it is likely that most of the optically observed subgrains have been through the growth stage described above. This is consistent with White's (1979) observation of two discrete "subgrain" sizes—smaller in TEM than in optical thin-section - and with the widespread description of similar sizes of optical "subgrains" and recrystallized grains (Hobbs, 1968; Bell and Etheridge, 1976). The local grain boundary migration simply results from its enhanced mobility at some critical misorientation, which may vary from boundary to boundary, together with the local increase in plastic strain energy due to increased sub-boundary misorientation. This local increase in strain energy is an intrinsic result of the subgrain rotation process, and whereas it provides some driving force for nucleation, it does not result in hardening. The "migration recrystallization" of Guillopé and Poirier (1979) presumably takes place when grain boundary mobility is high enough to allow grain growth to proceed at a faster rate than the development of a substructure and subsequent subgrain rotation. The boundary energy of the recrystallized grains is likely to provide a significant fraction of the driving force for this grain growth. 


\section{Continued deformation after substantial recrystallization}

In the Cazadero albite, substantially recrystallized regions consist of relatively equant grains of similar size to the nuclei discussed above, but they contain both free dislocations and loosely to well constructed sub-boundaries. It is possible that some or all of this substructure has been imposed in one or more minor straining events subsequent to the main deformation and recrystallization (White, 1977). However, the similarity of this substructure to the one that is transitional to the primary recrystallization stage (compare Figures 5 and 8) suggests that they formed under the same conditions, and that the intragranular substructure is a product of dynamic recrystallization and continuing deformation. In this event, it is important to try to understand the relative roles of dislocation flow processes and dynamic recrystallization in the continuing deformation. There are two main problems to be considered, first, the effect of dynamic recrystallization on strength, and, second, its contribution to fabric (texture) development.

The mechanically important question is whether the initial population of recrystallized grains persists during continued deformation, or whether continuous (or cyclic) recrystallization takes place. In the latter case, the strength of the recrystallized aggregate will be less than that of the unrecrystallized material (McQueen and Jonas, 1975; Sellars, 1978; Zeuch, 1982). However, retention of the initial population, with limited grain boundary migration, will not lead to significant softening. We do not have the critical evidence to distinguish between these two scenarios in the Cazadero albite, but some of our observations are at least consistent with retention of the initial grain population. For example, recrystallized grains with very low dislocation densities are rarely found, except at the onset of recrystallization, and no evidence of frozen-in, new nucleation stages was seen in the thoroughly recrystallized regions. We therefore suggest that continued deformation of the recrystallized aggregate could have taken place by dislocation flow within individual grains, and that the existing grain boundaries were sufficiently efficient sinks for mobile dislocations and sub-boundaries to remove the need for further recrystallization. Grain boundary migration took place but only where required to retain approximately equiaxed grainshapes (Bell and Etheridge, 1976; Etheridge and Kirby, 1983). The absence of significant softening under some experimental conditions, especially those involving subgrain rotation may be consistent with this microstructural model (Schmid et al., 1980; Kirby and Etheridge, 1983).

With respect to fabric (texture) development, recrystallized grains with initial orientations close to that of their deformed host (Hobbs, 1968; Ransom, 1971; Wilson, 1973; Bell and Etheridge, 1976) can be explained by the subgrain rotation model. These grains will continue to rotate throughout further 
deformation, but, since they are rotating largely in response to dislocation motion, their orientations will remain within the same fabric pattern as that of their deformed host grain(s). In both naturally and experimentally deformed minerals, fabric patterns of deformed original grains and their recrystallized products are generally indistinguishable (Ransom, 1971; Bell and Etheridge, 1976; Etheridge and Kirby, 1983), providing further support for this recrystallization mechanism. Any form of homogeneous nucleation, whether or not the nucleus orientation is stress-controlled (Avé Lallement and Carter, 1970), is likely to produce orientations outside the deformed grain fabric. A few such grains (Figure 6) are present in the albite, but continued deformation would rotate them back to that fabric, and the overall pattern would be essentially unchanged by recrystallization.

\section{CONCLUSIONS}

1) Dynamic recrystallization in the Cazadero albite took place by progressive rotation of subgrains. However, a discrete "nucleation" stage can be recognized, at which limited subgrain growth (or coalescence) took place, leading to an approximately twofold increase in grainsize when the misorientation reached a few degrees. Following this increase in size, deformation of the new grains took place by normal dislocation processes.

2) There does not appear to be a narrowly defined critical angle for the subgrain growth ("nucleation") stage of recrystallization. It can take place at values of misorientation between about $3^{\circ}$ and $5^{\circ}$, and presumably depends upon boundary orientation and shape, and the adjacent dislocation substructure. Detailed boundary structure within this angular range is also quite variable.

3) Except for rare, apparently homogeneously nucleated grains, all recrystallized grains are formed from a part of the deformed substructure, and all subsequent grain rotations are due to the migration of individual dislocations or dislocation walls, even in thoroughly recrystallized material. The fabric (texture) of the recrystallized material will thus be the same as that of the deformed original grains, unless substantial grain growth takes place.

\section{Acknowledgements}

The electron microscopy was carried out on the JEM200A instrument in the Department of Physics at Monash University, and Alec McLaren's interest and guidance is very much appreciated. J.D.F. was in receipt of a Commonwealth Postgraduate Scholarship at the time. We would also like to thank Bruce Hobbs for stimulating discussion, and Bob Bryant for his careful processing of the original TEM photographs. M.A.E. would like to acknowledge financial support from the Australian Research Grants Scheme, grants no. E75/15081, E78/15232. 


\section{References}

Aust, K. T. and Rutter, J. W. Trans. A.I.M.E. 215, 119 (1959).

Avé Lallement, H. G. and Carter, N. L. Bull. Geol. Soc. Amer., 81, 2203 (1970).

Bailey, J. E. and Hirsch, P. B. Proc. Roy. Soc. London. Ser. A, 267, 11 (1962).

Bell, T. H. and Etheridge, M. A. Tectonomphysics, 32, 235 (1976).

Clarebrough, L. M. and Forwood, C. T. phys. stat. sol. (a), 58, 597 (1980).

Coleman, R. G. and Lee, D. E. J. Petrology, 4, 260 (1963).

Edward, G. H., Etheridge, M. A. and Hobbs, B. E. Textures and Microstructures, 5, 127 (1982).

Ernst, W. G. Tectonophysics, 17, 255 (1973).

Etheridge, M. A. and Kirby, S. H. Tectonophysics, in press (1983).

Etheridge, M. A. and Wilkie, J. C. Tectonophysics, 58, 159 (1979).

Faivre, P. J. Appl. Cryst., 8, 356 (1975).

Gordon, P. and Vandemeer, R. A. In: H. Margolin (ed.) Recrystallization, Grain Growth and Textures. Am. Soc. Metals, 205-206 (1966).

Guillopé, M. and Poirier, J.-P. J. Geophys. Res., 84, 5557 (1979).

Hobbs, B. E. Tectonophysics, 6, 353 (1968).

Kirby, S. H. and Etheridge, M. A. Tectonophysics, in press (1983).

McQueen, H. J. and Jonas, J. J. In: R. J. Arsenault (ed.) Plastic Deformation of Materials. Treatise, Material Sci. Tech., 6, pp. 393-493 (1975).

Marshall, D. B. and McLaren, A. C. J. Material Sci., 12, 893 (1977).

Marshall, D. B., Vernon, R. H. and Hobbs, B. E. Contrib. Mineral. Petrol., 57, 49 (1976).

Marshall, D. B. and Wilson, C. J. L. Contrib. Mineral. Petrol., 57, 55 (1976).

Olsen, T. S. and Kohlstedt, D. L. Trans. Am. Geophys. Union EOS, 62, 395 (1981).

Poirier, J.-P. and Nicolas, A. J. Geol., 83, 707 (1975).

Pumphrey, P. H. and Bowkett, K. M. phys. stat. sol. (a), 2, 339 (1970).

Ransom, D. M. Mineral. Mag., 38, 83 (1971).

Schmid, S. M., Paterson, M. S. and Boland, J. N. Tectonophysics, 65, 245 (1980).

Sellars, C. M. Phil. Trans. Roy. Soc. London, Ser. A, 288, 147 (1978).

Takeuchi, S. and Argon, A. S. J. Material Sci., 11, 1542 (1976).

Taylor, H. P. and Coleman, R. G. Bull. Geol. Soc. Amer., 79, 1727 (1968).

Twiss, R. J. Pure Appl. Geophys., 115, 227 (1977).

Vogel, T. A. and Seifert, K. E. Amer. Mineral., 50, 514 (1965).

White, S. Nature, 244, 276 (1973).

White, S. Contrib. Mineral. Petrol., 50, 287 (1975).

White, S. Phil. Trans. Roy. Soc. London, Ser. A, 283, 69 (1976).

White, S. Tectonophysics, 39, 143 (1977).

White, S. Contrib. Mineral. Petrol., 70, 193 (1979).

Wilson, C. J. L. Tectonophysics, 19, 39 (1973).

Young, C. T., Steele, J. H. and Lytton, J. L. Met. Trans., 4, 2081 (1973).

Zeuch, D. H. Tectonophysics, 83, 293 (1982). 